\title{
Electrically Conductive Gels Prepared from Syndiotactic Polystyrene and an Ionic Liquid
}

Hideyuki Itagaki, *1,2 Naoto Yoshida, ${ }^{1}$ Takumi Sano, ${ }^{2}$ Mayu Yokoyama, ${ }^{1}$ Nozomi Iba, ${ }^{1}$

Ryotaro Sugiyama ${ }^{1}$ and Masakatsu Kuroki ${ }^{3}$

${ }^{1}$ Department of Chemistry, School of Education, Shizuoka University,

${ }^{2}$ Department of Chemistry, Graduate School of Science and Technology, Shizuoka University,

836 Ohya, Suruga-ku, Shizuoka 422-8529, Japan

${ }^{3}$ R \& D Laboratory, Taica Corporation, 789 Miyakami, Shimizu-ku, Shizuoka 424-0911, Japan

Tel: +81-54-238-4627, Fax: +81-54-237-3354, E-mail: itagaki.hideyuki@ shizuoka.ac.jp 


\section{Supporting data}

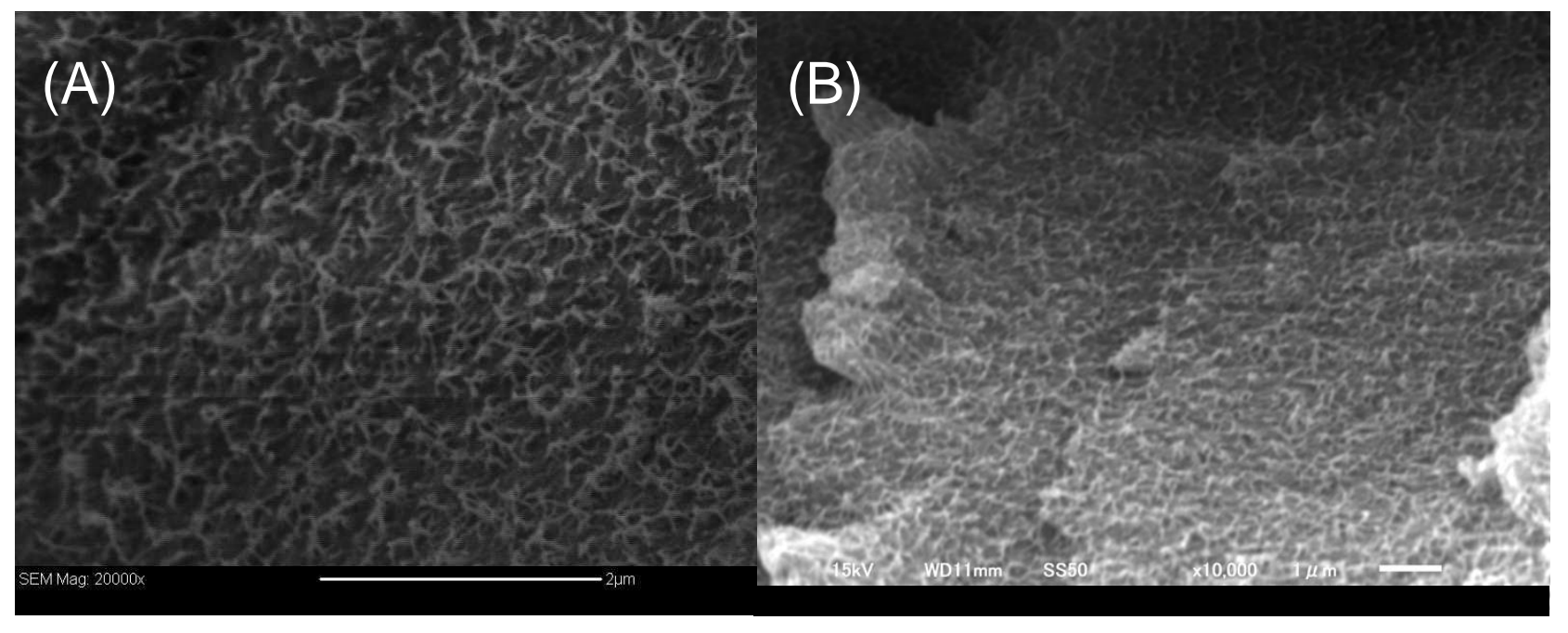

Figure S1.

SEM pictures of (A) dried SPS/Py gel (magnification, 20,000 fold) and (B) dried SPS/Py/[C4py]Br gel after washing with methanol (magnification, 10,000 fold). 
(A)

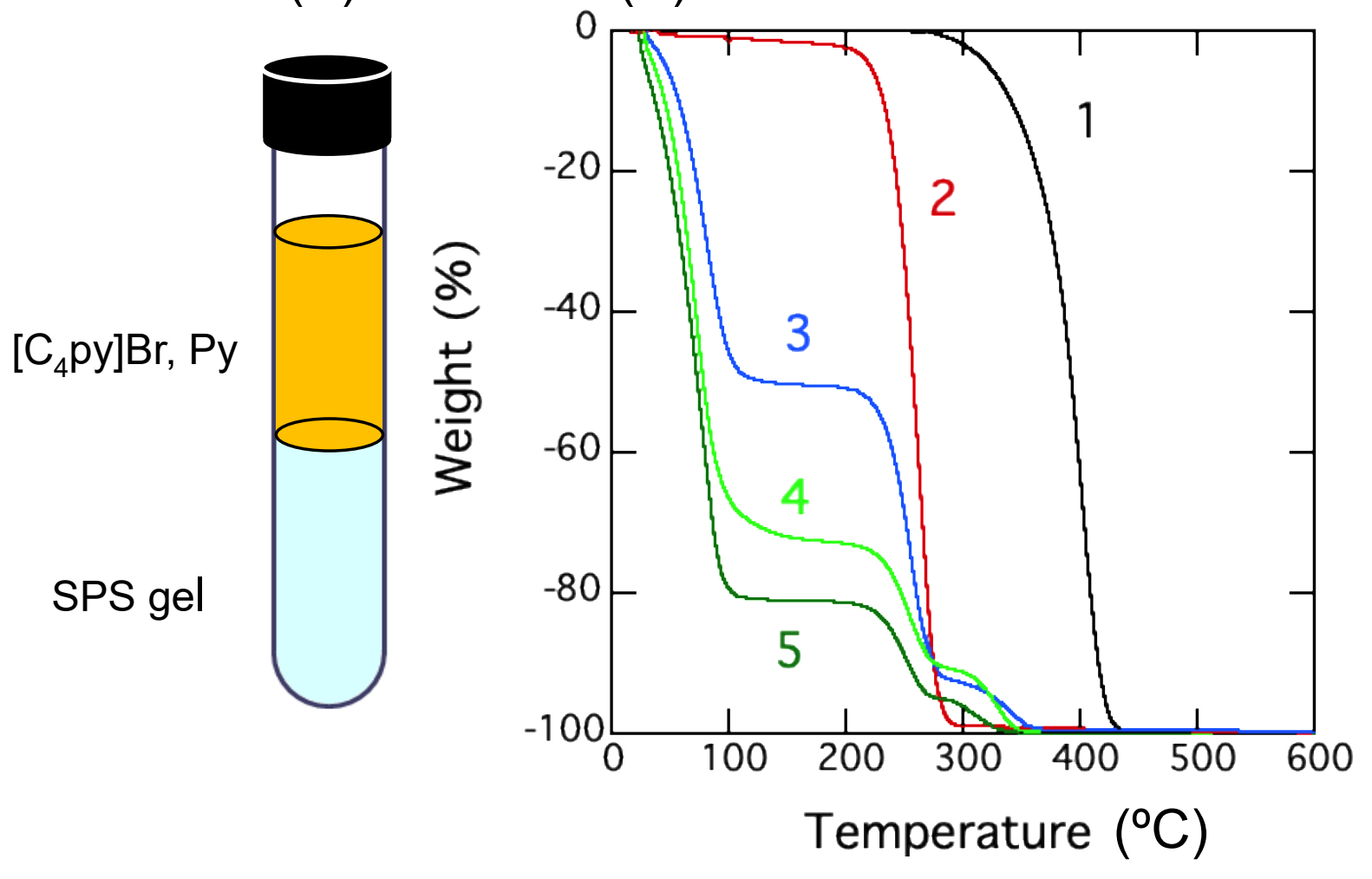

Figure S2.

(A) Image of how to mount [ $\left.\mathrm{C}_{4} \mathrm{py}\right] \mathrm{Br}$ or $\left[\mathrm{C}_{4} \mathrm{py}\right] \mathrm{Br} / \mathrm{Py}$ on an SPS gel. (B) Thermogravimetry traces of SPS/Py/[C ${ }_{4}$ py $] \mathrm{Br}$ gels at a scan rate of $10{ }^{\circ} \mathrm{C} / \mathrm{min}$ : 1 . SPS pellet, 2. ILBr, 3 5. SPS gels after the mount of ILBr or Py solutions of ILBr for 1 week on SPS/Py/[C 4 py]Br gels where SPS was 5 wt\% and the weight ratio between Py and ILBr was 9:1. The mounting solution was; 3. ILBr only, 4. $\mathrm{ILBr}: \mathrm{Py}=5: 5,5$. ILBr:Py=2:8 (weight ratio). 


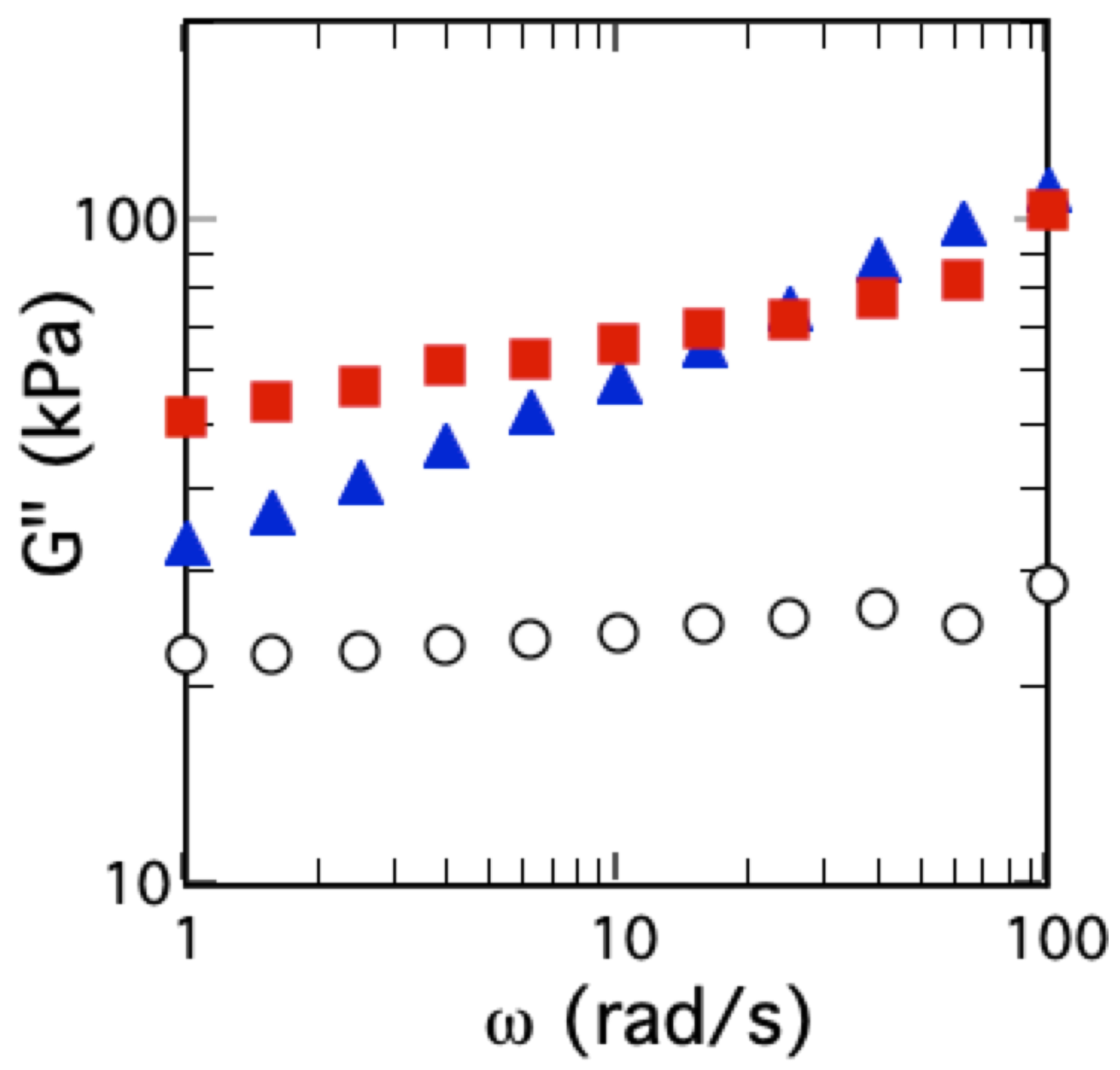

Figure S3.

Frequency dependence of the loss elastic modulus, G", of SPS/Py/[C $4 \mathrm{py}] \mathrm{Br}$ gel [the fractions of [C4py]Br were 35 (black circle) and $41 \mathrm{wt} \%$ (red square)] and SPS/Py gels (blue triangle) at $25{ }^{\circ} \mathrm{C}$ : the SPS concentrations of all the gels were $5 \mathrm{wt} \%$. 